\title{
Mamíferos silvestres predados por cães domésticos em fragmentos de Mata Atlântica no sul do Brasil
}

\author{
Alan Deivid Pereira ${ }^{1 *}$ \\ Matheus Henrique Antoniazzi ${ }^{1}$ \\ Ana Paula Vidotto-Magnoni ${ }^{2}$ \\ Mário Luís Orsi ${ }^{2}$ \\ ' Universidade Estadual de Londrina, Centro de Ciências Biológicas \\ Programa de Pós-Graduação em Ciências Biológicas \\ Área de Concentração: Biodiversidade e Conservação de Habitats Fragmentados \\ ${ }^{2}$ Universidade Estadual de Londrina, Centro de Ciências Biológicas \\ Rodovia Celso Garcia Cid, PR-445, Km 380, CEP 86.057-970, Londrina - PR, Brasil \\ * Autor para correspondência \\ alandeivid_bio@live.com
}

Submetido em 09/10/2018

Aceito para publicação em 06/04/2019

\section{Resumo}

O impacto de espécies exóticas sobre a fauna nativa está entre as maiores ameaças à biodiversidade global. O cão doméstico é considerado invasor quando acessa áreas naturais, podendo sobreviver independentemente da intervenção humana. No Brasil há poucos estudos com informações sobre o impacto da predação ou caça de cães sobre a fauna silvestre. Relatamos cinco eventos de predação de mamíferos silvestres por cães domésticos em fragmentos da Mata Atlântica na região norte do estado do Paraná, Brasil. Entre junho e setembro de 2018, espécimes de Didelphis albiventris (gambá-de-orelha-branca), Dasypus novemcinctus (tatu-galinha), Sapajus nigritus (macaco-prego) e Eira barbara (irara) foram abatidos por cão doméstico tanto em fragmentos de área urbana quanto de rural. Cães domésticos oferecem riscos à fauna silvestre inclusive àquelas espécies ameaçadas de extinção, sendo de extrema urgência ações diversificadas para eliminar e reduzir o acesso desses animais a fragmentos florestais. Nossos dados são um indicativo do impacto de cães domésticos sobre a fauna silvestre da Mata Atlântica no norte do estado do Paraná e podem dar suporte para medidas de controle, contenção e erradicação, obtendo-se assim a mitigação do impacto dos cães domésticos.

Palavras-chave: Canis lupus familiaris; Cão feral; Conservação; Espécie exótica; Predação

\section{Abstract}

Invasive domestic dogs prey on wild mammals in Atlantic Rainforest fragments of southern Brazil. Invasive exotic species impact native fauna adversely and are one of the leading causes of global biodiversity change. The domestic dog can be classified as an invasive species after its access to natural areas followed by their ability to survive independently of human intervention. In Brazil, the effects of dog predation and hunting on wildlife remains unclear. In this study, we report five wild-mammal predation events involving domestic dogs in fragments of the Brazilian Atlantic Forest (northern Paraná State, Brazil). Between June and September 2018, we found that specimens of Didelphis albiventris (white-eared opossum), Dasypus novemcinctus (nine-banded 
armadillo), Sapajus nigritus (black capuchin), and Eira barbara (tayra) were killed by domestic dogs in both urban and rural areas. Invasive domestic dogs can pose a risk to wildlife, especially to threatened species. We suggest that diversified measures be taken to impede and reduce the access of these animals to forest fragments. Furthermore, our findings indicate that control, containment, and eradication measures can reduce the negative effects of domestic dogs on wildlife.

Key words: Alien species; Canis lupus familiaris; Conservation; Feral dog; Predation

Espécies invasoras representam uma das ameaças mais significativas para a biodiversidade global e à funcionalidade dos ecossistemas e ainda podem causar prejuízos à economia, além de sérios riscos para a saúde humana (CLOUT; WILLIAMS, 2009). Muitas espécies não nativas invasoras tiveram a introdução mediada por ação humana, seja para fins de produção, consumo, comercialização ou uso como animais de estimação (i.e. cães, gatos e aves) (SCHÜTTLER; KAREZ, 2009). Nesse contexto, entre as espécies que vivem em intensa associação com humanos, Canis lupus familiaris Linnaeus, 1758 (cão comum) figura entre os animais domésticos mais difundidos por todo o mundo (HUGHES; MACDONALD, 2013).

O cão doméstico é considerado uma espécie invasora ou feral quando acessa áreas naturais, podendo sobreviver independentemente da assistência ou intervenção humana (BOITANI; CIUCCI, 1995; YOUNG et al., 2011). Tanto cães invasores quanto ferais podem afetar a fauna nativa através da predação, competição, perturbação, hibridização e transmissão de doenças (YOUNG et al., 2011). Nesse sentido, cães em áreas florestadas podem caçar animais silvestres por subsistência (HUGHES; MACDONALD, 2013; LESSA et al., 2016) ou caçar por diversão, ferindo ou matando animais silvestres, tomando presas tanto grandes quanto pequenas, e raramente consumindo suas vítimas (HUGHES; MACDONALD, 2013).

O impacto dos cães domésticos sobre a fauna silvestre ganha atenção dos conservacionistas nos últimos anos (YOUNG et al., 2011; HUGHES; MACDONALD, 2013; LESSA et al., 2016). No Brasil ainda são poucos os estudos que focam nas informações sobre o impacto da predação ou de ataques não letais de cães sobre a fauna silvestre (GALETTI; SAZIMA, 2006; CAMPOS et al., 2007; OLIVEIRA et al., 2008; SRBEK-ARAUJO; CHIARELLO, 2008; RANGEL et al., 2013; GATTI et al., 2018). Para a região sul do Brasil apenas o estado de Santa Catariana conta com registros de predação de animais silvestres por cães domésticos (GRAIPEL et al., 2001). Sendo assim, neste estudo são relatados cinco eventos de caça, por cães domésticos, de quatro mamíferos silvestres na porção Sul da Mata Atlântica brasileira, região norte do estado do Paraná.

Os registros ocorreram em fragmentos de Floresta Estacional Semidecidual de Mata Atlântica em meios urbanos e rurais, localizados na região do município de Londrina, norte do Paraná, Brasil (Figura 1). Todos os registros aqui apresentados foram obtidos por um dos autores, e as informações fornecidas pela comunidade local, sendo confirmados por fotos e observações oportunistas dos ataques. As coordenadas geográficas foram registras por GPS e projetadas para o Datum WGS84.

No dia 22 de junho de 2018, próximo ao Centro de Ciências Biológicas da Universidade de LondrinaPR (UEL) (2319'35.83’S, 51¹1'42.75’O, Figura 1), encontramos uma fêmea adulta de Didelphis albiventris Lund, 1840 com mordidas e desfigurações ao longo do corpo (Figura 2A). Funcionários da instituição confirmaram a predação do animal silvestre por cães que residem nas imediações do campus. No dia 19 de julho de 2018, na região rural de Londrina (2336'45.9'S, $51^{\circ} 05^{\prime} 57.3$ 'O, Figura 1), registramos um jovem macho de Dasypus novemcinctus Linnaeus, 1758 com muitos sinais de mordidas recentes ao longo do corpo (Figura 1B). O abatimento por cães foi confirmado por um morador que reside na localidade. $\mathrm{O}$ terceiro registro ocorreu em 21 de julho de 2018, no Campus da UEL (2319’34.29”'S, 51¹2'6.34’O, Figura 1). Um indivíduo jovem macho de Sapajus nigritus (Goldfuss, 1809) foi caçado por um cão sem coleira nas imediações do campus universitário. Funcionários relataram a perseguição de abatimento do macaco-prego pelo cão 
FIGURA 1: Localização dos registros de morte de animais silvestres por cães domésticos em fragmentos urbanos e rurais da Floresta Estacional Semidecidual (Mata Atlântica) na região de Londrina, norte do Paraná, Brasil. PEMG = Parque Estadual Mata dos Godoy. Letras representam os fragmentos onde ocorreram os registros, sendo: A = Campus da Universidade Estadual de Londrina; $\mathrm{B}=$ Horto UEL; $\mathrm{C}=$ Fragmento Patrimônio Regina; $\mathrm{D}=$ Fragmento Lerrovile.

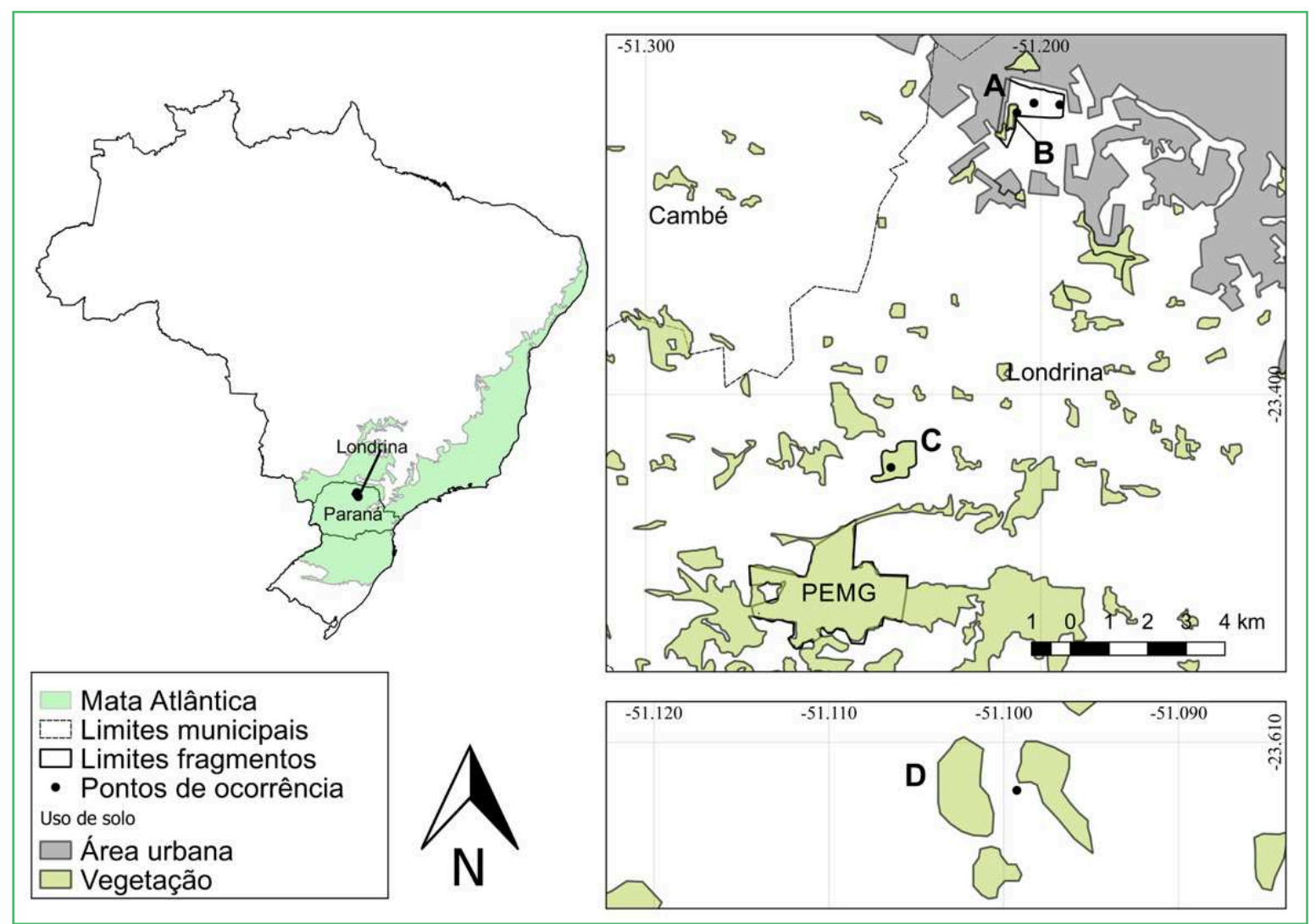

doméstico (Figura 2C). Em 20 de agosto de 2018,

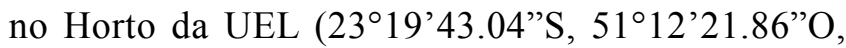
Figura 1), encontramos um macho adulto de $D$. albiventris com sinais de mordidas ao longo corpo, uma funcionária da instituição confirmou o abatimento do animal por cães que residem no campus (Figura 2D). O ultimo registro ocorreu em 12 de setembro de 2018, na área rural de Londrina, próximo ao Parque Estadual Mata dos Godoy (2325'6.70’'S, 51¹4'16.17’O, Figura 1). Registramos um jovem macho de Eira barbara (Linnaeus, 1758) abatido por cães, o registro foi fornecido por um morador residente do local (Figura 2E).

Os registros apresentados neste estudo reforçam a necessidade de estudos mais abrangentes que avaliem os impactos de animais domésticos (exóticos) sobre a fauna silvestre no Brasil, uma vez que em um curto período de tempo e sem um método amostral padronizado registramos ataques letais de cães sobre a fauna selvagem em uma região de Mata Atlântica altamente fragmentada. $\mathrm{Na}$ revisão de Hughes e MacDonald (2013), foram listadas 64 espécies de animais silvestres com algum tipo de interação com cães domésticos ou ferais em 29 países em todo o mundo. Para o Brasil, Lessa et al. (2016) encontraram 37 espécies de animais silvestres interagindo (predação, competição e transmissão de doenças) com cães. Desse total, 85\% (27) são mamíferos de médio ou grande porte, sendo que 19 (55\%) estão listados como ameaçados Brasil (LESSA et al., 2016).

Poucos trabalhos no Brasil avaliaram de fato a predação de animais silvestres por cães, entre eles Campos et al. (2007), ao analisarem 137 fezes de cães, coletadas em fragmentos florestais, constataram que $65,38 \%$ dos itens encontrados são de origem animal, indicando um alto nível de predação de vida selvagem por cães, sendo o quati o mais presente nas fezes. Assim como neste estudo, a maioria dos dados 
FIGURA 2: Carcaça dos animais abatidos por cães na região da Mata Atlântica norte do Paraná, Brasil, sendo: (A e D) Didelphis albiventris; (B) Dasypus novemcinctus; (C) Sapajus nigritus; (E) Eira barbara.
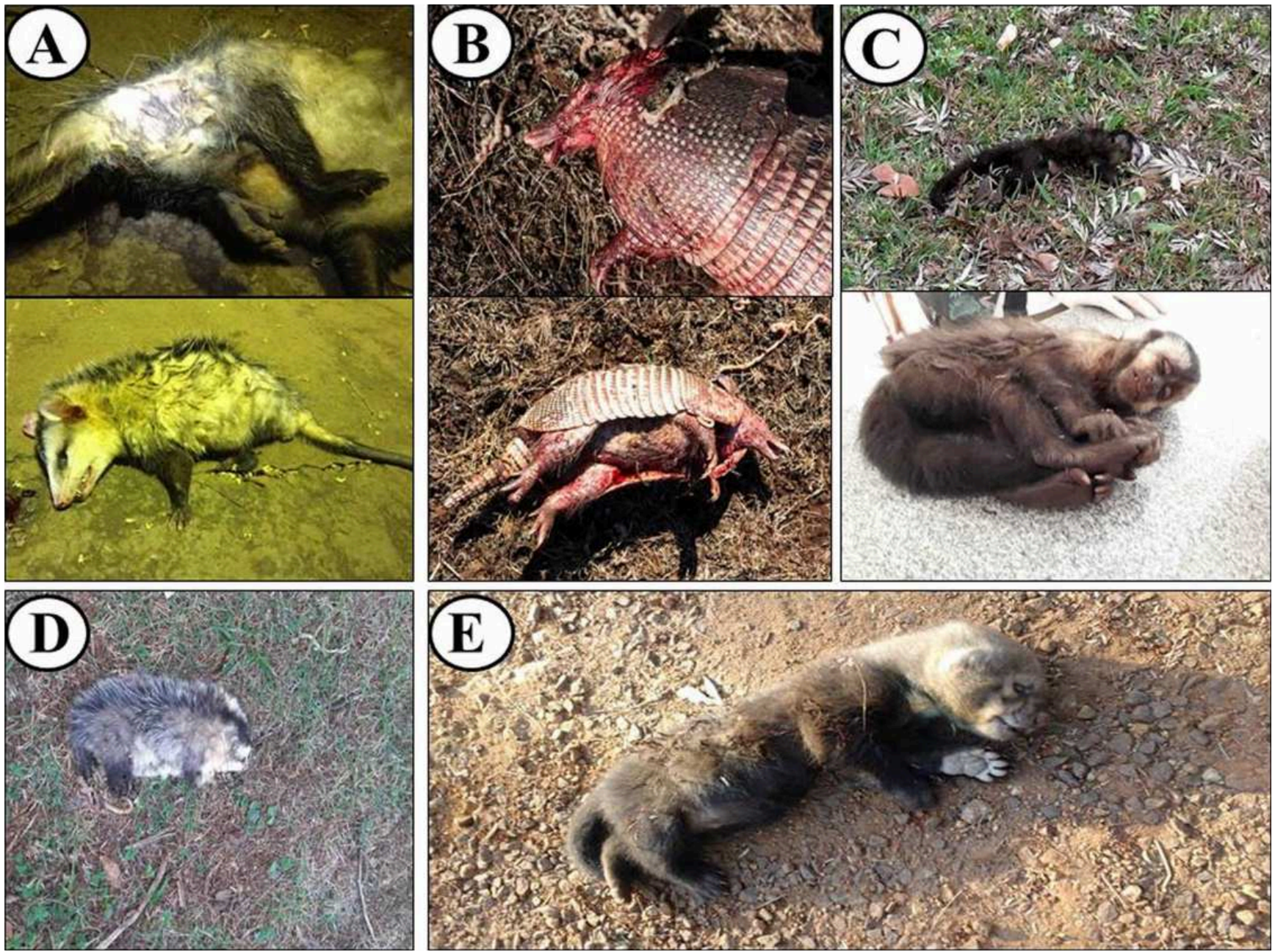

disponíveis para o Brasil está relacionada ao abate ou à caça de animais silvestres por cães (GALETTI; SAZIMA, 2006; OLIVEIRA et al., 2008, RANGEL et al., 2013; GATTI et al., 2018). Entre os registros do presente estudo, E. barbara não constava na lista de espécies abatidas por cão doméstico, sendo este o primeiro registro para o Brasil; as demais espécies já são listadas na literatura (Tabela 1). A lista de animais silvestres que são atacados por cães pode variar de pequenos anfíbios (sapos), répteis (lagartos) e aves a alguns mamíferos de médio porte e grande porte (tatus, macacos, veados, tapitis, preás, pacas, porcos espinho e até mesmo antas) (Tabela 1).

A maioria dos dados referentes ao impacto de cães invasores sobre a fauna silvestre é pertencente à região da Mata Atlântica, indicando uma alta proporção de cães em remanescentes florestais, muitos deles sendo áreas de proteção (SRBEK-ARAÚJO; CHIARELLO, 2008; LESSA et al., 2016; SILVA et al., 2018). Nossos dados indicam o impacto de cães domésticos sobre a fauna silvestre da região norte do estado do Paraná, pois em um período menor que seis meses registramos quatro espécies diferentes de mamíferos abatidos por cães tanto em ambientes urbanos quanto na zona rural. Conforme Silva-Rodríguez e Sieving (2011), cães domésticos tendem a caçar animais nativos em função do seu instinto e, por causa da perseguição, cães podem afetar o comportamento e sucesso reprodutivo desses animais (SILVA-RODRÍGUEZ; SIEVING, 2011). 
TABELA 1: Lista das espécies de vertebrados silvestres com registro de predação por cão doméstico em território brasileiro citadas na literatura.

\begin{tabular}{|c|c|c|c|c|}
\hline \multirow{2}{*}{ Espécies } & \multirow{2}{*}{ Local } & \multicolumn{2}{|c|}{ Coordenadas } & \multirow{2}{*}{ Ref* } \\
\hline & & Latitude & Longitude & \\
\hline \multicolumn{5}{|l|}{ ANFÍBIOS } \\
\hline Leptodactylus labyrinthicus & Campinas-SP & -22.8215 & -47.1102 & 1 \\
\hline \multicolumn{5}{|l|}{ RÉPTEIS } \\
\hline Tupinambis merianae & Campinas-SP & -22.8215 & -47.1102 & 1 \\
\hline Salvator merianae & Rio de Janeiro-RJ & -22.9688 & -43.2259 & 3 \\
\hline \multicolumn{5}{|l|}{ AVES } \\
\hline Nyctidromus albicollis & Campinas-SP & -22.8215 & -47.1102 & 1 \\
\hline Crax blumenbachii & Cachoeiras de Macacu-RJ & -22.4519 & -42.7710 & 5 \\
\hline Hydropsalis albicollis & Sem informação & - & - & 7 \\
\hline \multicolumn{5}{|l|}{ MAMÍFEROS } \\
\hline \multirow{4}{*}{ Didelphis aurita } & Campinas-SP & -22.8215 & -47.1102 & 1 \\
\hline & Florianópolis - SC & -27.7283 & -48.5332 & 6 \\
\hline & Rio de Janeiro & -22.9688 & -43.2259 & 1 \\
\hline & Londrina-PR & -23.3266 & -51.1952 & 9 \\
\hline \multirow{2}{*}{ Didelphis albiventris } & Londrina-PR & -23.3286 & -51.2060 & 9 \\
\hline & Piracicaba-SP & -22.7 & -47.6333 & 8 \\
\hline Lutreolina crassicaudata & Piracicaba-SP & -22.7 & -47.6333 & 8 \\
\hline Metachirus nudicaudatus & Sem informação & - & - & 7 \\
\hline Philander frenatus & Sem informação & - & - & 7 \\
\hline \multirow[t]{2}{*}{ Tamandua tetradactyla } & Rio de Janeiro-RJ & -22.9688 & -43.2259 & 3 \\
\hline & Campinas-SP & -22.8215 & -47.1102 & 1 \\
\hline \multirow[t]{2}{*}{ Dasypus novemcinctus } & Londrina-PR & -23.6127 & -51.0992 & 9 \\
\hline & Piracicaba-SP & -22.7 & -47.6333 & 8 \\
\hline Dasypus septemcinctus & Sem informação & - & - & 7 \\
\hline Euphractus sexcintus & Sem informação & - & - & 7 \\
\hline Tapirus terrestris & Pinheiros-ES & -18.3429 & -40.1445 & 4 \\
\hline Mazama gouazoubira & Campinas-SP & -22.8215 & -47.1102 & 1 \\
\hline \multirow[t]{2}{*}{ Alouatta guariba } & Campinas-SP & -22.8215 & -47.1102 & 1 \\
\hline & Campinas-SP & -22.8215 & -47.1102 & 1 \\
\hline \multirow[t]{2}{*}{ Sapajus nigritus } & Bom Jesus do Madeira-MG & -20.7186 & -42.4807 & 2 \\
\hline & Londrina-PR & -23.3261 & -51.2017 & 9 \\
\hline Eira barbara & Londrina-PR & -23.4185 & -51.2378 & 9 \\
\hline Galictis cuja & Piracicaba-SP & -22.7 & -47.6333 & 8 \\
\hline Nasua nasua & Piracicaba-SP & -22.7 & -47.6333 & 8 \\
\hline Procyon cancrivorus & Rio de Janeiro & -22.9688 & -43.2259 & 3 \\
\hline \multirow[t]{2}{*}{ Sylvilagus brasiliensis } & Campinas-SP & -22.8215 & -47.1102 & 1 \\
\hline & Campinas-SP & -22.8215 & -47.1102 & 1 \\
\hline \multirow[t]{2}{*}{ Cavia aperea } & Sem informação & - & - & 7 \\
\hline & Piracicaba-SP & -22.7 & -47.6333 & 8 \\
\hline Calomys tener & Piracicaba-SP & -22.7 & -47.6333 & 8 \\
\hline Hydrochoerus hydrochaeris & Sem informação & - & - & 7 \\
\hline
\end{tabular}




\begin{tabular}{lcccc}
\hline \multicolumn{1}{c}{ Espécies } & \multirow{2}{*}{ Local } & \multicolumn{2}{c}{ Coordenadas } & \multirow{2}{*}{ Ref* } \\
\cline { 3 - 4 } Cuniculus paca & Campinas-SP & -22.8215 & -47.1102 & 1 \\
Dasyprocta leporina & Sem informação & - & - & 7 \\
Myocastor coypus & Sem informação & - & - & 7 \\
Coendou prehensilis & Piracicaba-SP & -22.7 & -47.6333 & 8 \\
Coendou spinosus & Piracicaba-SP & -22.7 & -47.6333 & 8 \\
Sciurus aestuans & Campinas-SP & -22.8215 & -47.1102 & 1 \\
\hline
\end{tabular}

Ref* = 1. Galetti e Sazima (2006); 2. Oliveira et al. (2008); 3. Rangel e Neiva (2013); 4. Gatti et al. (2018); 5. Bernardo (2012); 6. Graipel et al. (2001); 7. Brito et al. (2004); 8. Campos et al. (2007); 9. Presente estudo.

A maioria dos registros ocorreu em regiões periurbanas, tais regiões são caracterizadas como zonas de transição entre o espaço urbano e espaços rurais (VALE; GERARDI, 2006). Diferentemente de zonas rurais, em regiões urbanas muitas vezes o número de cães excede o número de humanos (HUGHES; MACDONALD, 2013). Em todos os casos não houve interferência direta de humanos, ou seja, com intenção e incentivo para os cães perseguirem os animais silvestres. Contudo, muitas vezes tratava-se de animais que residiam em habitações próximas a fragmentos, tendo livre acesso aos mesmos, ou seja, sem os devidos cuidados para evitar as fugas, caracterizando assim uma negligência por parte dos humanos responsáveis. Quanto menos cuidados animais domésticos receberem de seus donos, maior a probabilidade de eles predarem ou se alimentarem de animais selvagens para obterem proteínas para suplementar sua dieta (BUTLER; BIRGHAM, 2000; SILVA et al., 2018). Esse padrão é prevalente nos países em desenvolvimento porque a maioria dos animais domésticos é criada livre e não recebe cuidados adequados de saúde e alimentação (SILVA-RODRÍGUEZ; SIEVING, 2011).

No Brasil, somente em 2009, foi promulgada a resolução $n^{\circ} 05$ do Conselho Nacional do Meio Ambiente, denominada Estratégia nacional para espécies exóticas invasoras e apenas em 2018 foi regulamentada. E pela primeira vez, foi inserida a necessidade de manejo de fauna não nativa e invasora, a qual causa danos severos à biodiversidade. Mas ainda se faz necessário que outras medidas legais sejam tomadas nas esferas estaduais e municipais, como uma política efetiva sobre a conservação da fauna nativa, e, entre essas medidas, um plano de manejo com as espécies invasoras de alto impacto como os cães domésticos. Além do manejo de animais exóticos em unidades de conservação, fazem-se necessárias ações de educação ambiental para conscientização da população sobre a guarda responsável, vacinação e castração de seus animais domésticos, principalmente em áreas periurbanas, onde acreditamos que o problema é mais grave devido à alta abundância de cães domésticos em estado de abandono.

Nossos dados podem dar suporte para estudos mais abrangentes sobre o impacto de cães domésticos sobre a fauna silvestre em áreas urbanas. Ainda são escassos os registros como os deste estudo para o Brasil, o que inviabiliza identificar um padrão para esse tipo de interação. Acreditamos que novos estudos devem ser conduzidos para complementar o banco de dados de ataque de cães sobre a fauna silvestre. Como a maior parte desses registros são oportunísticos, a criação de um aplicativo acessível à comunidade científica e à população geral ajudaria a organizar e quantificar dados referentes à biomassa, ao total de espécies e ao número de animais abatidos por cães, tanto em unidades de conservação quanto em fragmentos urbanos.

\section{Agradecimentos}

O presente trabalho foi realizado com apoio da Coordenação de Aperfeiçoamento de Pessoal de Nível Superior (CAPES) - Código de Financiamento 1689817. 
Agradecemos aos funcionários do Horto da UEL e aos moradores locais que colaboraram na obtenção desses registros.

\section{Referências}

BERNARDO, C. S. S. Reintroduction as a conservation tool for threatened Galliformes: the Red-billed Curassow Crax blumenbachii case study from Rio de Janeiro state, Brazil. Journal of Ornithology, Berlin, v. 153, p. 135-140, 2012.

BOITANI, L.; CIUCCI, P. Comparative social ecology of feral dogs and wolves. Ethology Ecology \& Evolution, Firenze, v. 7, p. 4972, 1995.

BRITO, D.; OLIVEIRA, L.; MELLO, M. A. R. An overview of mammalian conservation at Poço das Antas Biological Reserve, southeastern Brazil. Journal for Nature Conservation, Tilburg, v. 12, p. 219-228, 2004.

BUTLER, J. R. A.; BIRGHAM, J. Demography and dog-human relationships of the dog population in Zimzabwean communal lands. Veterinary Record, London, v. 147, n. 16, p. 442-446, 2000.

CAMPOS, C. B.; ESTEVES, C. F.; FERRAZ, K. M. P. M. B.; CRAWSHAW, P. G.; VERDADE, L. M. Diet of free-ranging cats and dogs in a suburban and rural environment, south-eastern Brazil. Journal of Zoology, London, v. 273, n. 1, p. 14-20, 2007.

CLOUT, M. N.; WILLIAMS, P. A. Invasive species management: a handbook of principles and techniques. Oxford: Oxford University Press, 2009. $336 \mathrm{p}$.

GALETTI, M.; SAZIMA, I. Impacto de cães ferais em um fragmento urbano de Floresta Atlântica no sudeste do Brasil. Natureza \& Conservação, Curitiba, v. 4, n. 1, p. 58-63, 2006.

GATTI, A.; SEIBERT, J. B.; MOREIRA, D. O. A predation event by free-ranging dogs on the lowland tapir in the Brazilian Atlantic Forest. Animal Biodiversity and Conservation, Barcelona, v. 2, p. 2016-2019, 2018.

GRAIPEL, M. E., CHEREM, J. J.; XIMENEZ, A. Mamíferos terrestres não voadores da Ilha de Santa Catarina, sul do Brasil. Biotemas. Florianópolis, v. 14, n. 2, p. 109-140, 2001.

HUGHES, J.; MACDONALD, D. W. A review of the interactions between free-roaming domestic dogs and wildlife. Biological Conservation, Boston, v. 157, p. 341-351, 2013.
LESSA, I.; GUIMARÃES, T. C. S.; BERGALLO, H. G.; CUNHA, A.; VIEIRA, E. M. Domestic dogs in protected areas: a threat to Brazilian mammals? Natureza \& Conservação, Curitiba, v. 14, n. 2, p. 46-56, 2016.

OLIVEIRA, V. B.; LiNARES, A. M.; CORRÊA, G. L. C.; CHIARELLO, A. G. Predation on the black capuchin monkey Cebus nigritus (Primates: Cebidae) by domestic dogs Canis lupus familiaris (Carnivora: Canidae), in the Parque Estadual Serra do Brigadeiro, Minas Gerais, Brazil. Revista Brasileira de Zoologia, Curitiba, v. 25, n. 2, p. 376-378, 2008.

RANGEL, C. H.; HELENA, C.; BUNN, M. Predação de vertebrados por cães Canis lupus familiaris (Mammalia: Carnivora) no Jardim Botânico do Rio de Janeiro, RJ, Brasil. Biodiversidade Brasileira, Brasília, v. 3, n. 2, p. 261-269, 2013.

SRBEK-ARAUJO, A.; CHIARELLO, A. Domestic dogs in Atlantic forest preserves of south-eastern Brazil: a camera-trapping study on patterns of entrance and site occupancy rates. Brazilian Journal of Biology, São Carlos, v. 68, n. 4, p. 771-779, 2008.

SCHÜTTLER, E.; KAREZ, C. S. Especies exóticas invasoras en las Reservas de Biosfera de América Latina y el Caribe. Un informe técnico para fomentar el intercambio de experiencias entre las Reservas de Biosfera y promover el manejo efectivo de las invasiones biológicas. Montevideo: UNESCO, 2009. 304 p.

SILVA, K. V.K.A.; KENUP, C. F.; KREISCHER, C.; FERNANDEZ, F. A. S.; PIRES, A. S. Who let the dogs out? Occurrence, population size and daily activity of domestic dogs in an urban Atlantic Forest reserve. Perspectives in Ecology and Conservation, São Paulo, v. 16, n. 4, p. 228-233, 2018.

SILVA-RODRÍGUEZ, E. A.; SIEVING, K. E. Influence of care of domestic carnivores ontheir predation on vertebrates. Conservation Biology, San Francisco, v. 25, n. 4, p. 808-815, 2011.

VALE, A. R; GERARDI, L. H. O. Crescimento urbano e teorias sobre o espaço periurbano: analisando o caso do município de Araraquara (SP). In: GERARDI, L. H .O; CARVALHO, P. F. (Ed.). Geografia: ações e reflexões. Rio Claro: UNESP/IGCE, AGETEO, 2006. p. 231-246.

YOUNG, J. K.; OLSON, K. A.; READING, R. P.; AMGALANBAATAR, S.; BERGER, J. Is wildlife going to the dogs? Impacts of feral and free-roaming dogs on wildlife populations. BioScience, Berkeley, v. 61, n. 2, p. 125-132, 2011. 